hep-ph/0409271

\title{
Coarse-graining 1/2 BPS geometries of type IIB supergravity
}

\author{
Alex Buchel \\ Perimeter Institute for Theoretical Physics \\ Waterloo, Ontario N2J 2W9, Canada \\ Department of Applied Mathematics \\ University of Western Ontario \\ London, Ontario N6A 5B7, Canada
}

\begin{abstract}
Recently Lin, Lunin and Maldacena (LLM) [1] explicitly mapped 1/2 BPS excitations of type IIB supergravity on $A d S_{5} \times S^{5}$ into free fermion configurations. We discuss thermal coarse-gaining of LLM geometries by explicitly mapping the corresponding equilibrium finite temperature fermion configuration into supergravity. Following Mathur conjecture, a prescription of this sort should generate a horizon in the geometry. We did not find a horizon in finite temperature equilibrium LLM geometry. This most likely is due to the fact that coarse-graining is performed only in a half-BPS sector of the full Hilbert space of type IIB supergravity. For temperatures much less than the $A d S$ curvature scale the equilibrium background corresponding to nearly degenerate dual fermi-gas is found analytically.
\end{abstract}

September 2004 


\section{Introduction}

In recent years motivated and guided by the AdS/CFT correspondence [2], new lessons have been learned about time-like singularities and their resolution in supergravity and string theory. Firstly, the goal of exhibiting new tests of the duality has led to the construction of many new exact supergravity backgrounds. This includes metrics on non-compact Calabi-Yau spaces with and without fluxes, $G_{2}$-holonomy metrics, etc. Secondly, the correspondence with gauge theory has hinted at the correct interpretation and resolution of singularities. The mechanisms include confinement, chiral symmetry breaking, generation of a mass gap, etc. These recent advances have complemented the existing knowledge about singularities from closed strings and D-branes. On the contrary, a space-like singularity, such as behind the horizon of the Schwarzschild black hole or the Big Bang singularity in cosmology, is poorly understood.

An interesting approach towards understanding space-like singularities behind black hole horizons is initiate by Mathur and collaborators [3-7]. The implication of the proposal is that these singularities are not physical: the long-suspected picture of a 'stringy' Schwarzschild horizon with a smooth region inside it ${ }^{1}$ is just an artifact of a classical analysis. Rather, the correct physical picture is akin the one arising in statistical mechanics. Much like equilibrium thermodynamics arises from the thermal coarse-graining over an ensemble of microstates of a dynamical system, the black hole solution (with a Schwarzschild horizon) is a result of a thermal averaging over its true microstate-geometries. These microstate-geometries are precisely the ones that the Bekenstein-Hawking entropy accounts for. Each one of the black hole microstates represents a smooth, singularity and horizon-free geometry. Currently the detailed understanding of black hole microstates exists for a rather degenerate case: a supersymmetries D1-D5 system in Ramond ground state. This black hole has zero temperature and zero macroscopic area of the horizon. In the last couple months very encouraging results appear [8-14] that suggest that Mathur's program of explaining the microstates of a black hole can be extended to a three-charge brane configuration, which, though supersymmetric, has a finite horizon area.

Understanding the microstates of a non-extremal black hole is a daunting task. For one reason, it is not even clear that all such states allow for a gravitational description ${ }^{2}$. But even if identified, a prescription has to be formulated how to coarse-grain these

\footnotetext{
${ }^{1}$ Having only the local large curvature region of the geometry resolved by the string corrections.

${ }^{2}$ Some microstates might not be even geometrical.
} 
micro-geometries. Such a prescription is evident in case there is a dual, purely field theoretical, formulation of geometries: all we have to do it to form a canonical (or grand canonical) ensemble of the dual field theory and map resulting thermal equilibrium configuration into the gravity. It is natural to conjecture that this procedure would produce a geometry with a Schwarzschild horizon. We would like to stress that above conjecture (as well as Mathur's interpretation of a black hole) is a very intuitive extension of the finite temperature gauge theory/string theory correspondence [15] where (in the simplest case) a large Schwarzschild black hole in global $A d S_{5}$ geometry corresponds to equilibrium finite temperature $\mathcal{N}=4 S U(N)$ supersymmetric Yang-Mills (SYM) theory on $S^{3} \times R$.

In [1] (LLM) the authors explicitly constructed smooth (and without horizon) type IIB supergravity backgrounds holographically dual to chiral primary operators of $\mathcal{N}=$ 4 SYM. These are operators with conformal weight $\triangle=J$, where $J$ is a particular $U(1)$ charge of the R-symmetry group. The LLM geometries are a subset of microgeometries one would have to use to realize a non-extremal black hole solution ${ }^{3}$ with $A d S_{5}$ asymptotic. In this paper we discuss thermal coarse-graining of LLM geometries. This is achieved by mapping the finite temperature free fermion description $[17,1]$ of $1 / 2$ BPS states of [1] into supergravity. The resulting thermally equilibrium supergravity background does not have a horizon. This is perhaps not surprising: one expects to recover the AdS-Schwarzschild solution upon thermal averaging over the whole Hilbert space of supergravity (string?) excitations in $A d S_{5} \times S^{5}$ rather than just over its $1 / 2$ BPS sector. Nonetheless, it is interesting to explore if supergravity backgrounds obtained in such a coarse-graining exhibit thermodynamic properties. We discuss this in a future publication.

The paper is organized as follows. In the next section we briefly review the LLM geometries. Using the free fermion description of LLM supergravity states we explain how to implement their finite temperature coarse-graining. In the limit of nearly degenerate fermi gas the dual geometry can be found analytically. We conclude in section 3.

\footnotetext{
${ }^{3}$ This black hole must have a chemical potential conjugate to the $U(1)$ R-symmetry charge. Thermodynamics of such a black hole in relation to a string dual of a large R-charge sector of SYM was discussed in [16].
} 


\section{LLM 1/2 BPS geometries at finite temperature}

Type IIB supergravity backgrounds dual to $1 / 2$ BPS states of $\mathcal{N}=4 S U(N)$ SYM on $S^{3} \times R[1]$ are characterized by a single function in $R^{2}:\left\{x_{1}, x_{2}\right\}$

$$
\begin{aligned}
z \equiv z\left(x_{1}, x_{2}, 0\right) & =1, & & \left\{x_{1}, x_{2}\right\} \in \mathcal{D} \subset R^{2}, \\
z & =0, & & \text { otherwise. }
\end{aligned}
$$

The $R^{2}$ in question has an interpretation as a phase space of (dual) free fermions [17], and $\mathcal{D}$ is a domain in this phase space occupied by a fermion droplet realizing a given chiral primary of SYM. The area $\mathcal{A}(\mathcal{D})$ of a droplet is quantized in units of

$$
\hbar \equiv 2 \pi \ell_{p}^{4}=2 \pi g_{s}\left(\alpha^{\prime}\right)^{2}
$$

so that

$$
\frac{\mathcal{A}(\mathcal{D})}{2 \pi \hbar}=N
$$

is the total 5 -form flux of the supergravity background. The quantum energy ${ }^{4}$ of a fermion at $\left(x_{1}, x_{2}\right)$ in the phase space is

$$
\epsilon \equiv \epsilon\left(x_{1}, x_{2}\right)=\frac{x_{1}^{2}+x_{2}^{2}}{2 \hbar} \equiv \frac{r^{2}}{2 \hbar} .
$$

A Fermi surface of zero temperature fermion configuration is a circle of radius

$$
r_{0}^{(T=0)}=(2 \hbar N)^{1 / 2},
$$

about the origin in the phase space. The energy of a particular fermion droplet $\mathcal{D}$ above the energy of the ground state is identified with the conformal dimension $\triangle$ of the SYM chiral primary, or an ADM mass of the corresponding supergravity background

$$
\begin{aligned}
\triangle=J & =\frac{1}{16 \pi^{3} \ell_{p}^{8}}\left[\int_{\mathcal{D}} d^{2} x\left(x_{1}^{2}+x_{2}^{2}\right)-\frac{1}{2 \pi}\left(\int_{\mathcal{D}} d^{2} x\right)^{2}\right] \\
& =\int_{\mathcal{D}} \frac{d^{2} x}{2 \pi \hbar} \frac{\frac{1}{2}\left(x_{1}^{2}+x_{2}^{2}\right)}{\hbar}-\frac{1}{2}\left(\int_{\mathcal{D}} \frac{d^{2} x}{2 \pi \hbar}\right)^{2} .
\end{aligned}
$$

Thus, zero temperature configuration (2.5) corresponds to $\triangle=0$, i.e., 'vacuum' (or $A d S_{5} \times S^{5}$ ) configuration

$$
\triangle^{(T=0)}=\int_{0}^{r_{0}} \frac{2 \pi r d r}{2 \pi \hbar} \frac{r^{2}}{2 \hbar}-\frac{1}{2}\left(\frac{\pi r_{0}^{2}}{2 \pi \hbar}\right)^{2}=0 .
$$

\footnotetext{
${ }^{4}$ In units of $1 / R_{A d S_{5}}$.
} 
Given $z\left(x_{1}, x_{2}, 0\right)$ of $(2.1)$ the corresponding supergravity background is [1]

$$
\begin{aligned}
d s^{2} & =-h^{-2}\left(d t+V_{i} d x^{i}\right)^{2}+h^{2}\left(d y^{2}+d x^{i} d x^{i}\right)+y e^{G} d \Omega_{3}^{2}+y e^{-G} d \tilde{\Omega}_{3}^{2}, \\
h^{-2} & =2 y \cosh G, \\
y \partial_{y} V_{i} & =\epsilon_{i j} \partial_{j} z, \quad y \partial_{i} V_{j}=\epsilon_{i j} \partial_{y} z, \\
z & =\frac{1}{2} \tanh G, \\
F_{(5)} & =F_{\mu \nu} d x^{\mu} \wedge d x^{\nu} \wedge d \Omega_{3}+\tilde{F}_{\mu \nu} d x^{\mu} \wedge d x^{\nu} \wedge d \tilde{\Omega}_{3}, \\
F & =d B_{t} \wedge(d t+V)+B_{t} d V+d \hat{B}, \\
\tilde{F} & =d \tilde{B}_{t} \wedge(d t+V)+\tilde{B}_{t} d V+d \tilde{\hat{B}}, \\
B_{t} & =-\frac{1}{4} y^{2} e^{2 G}, \quad \tilde{B}_{t}=-\frac{1}{4} y^{2} e^{-2 G}, \\
d \hat{B} & =-\frac{1}{4} y^{3} \star_{3} d\left(\frac{z+\frac{1}{2}}{y^{2}}\right), \quad d \tilde{\hat{B}}=-\frac{1}{4} y^{3} \star_{3} d\left(\frac{z-\frac{1}{2}}{y^{2}}\right),
\end{aligned}
$$

where $i=1,2$ and $\star_{3}$ is the flat space Hodge dual in the three dimensions parameterized by $y, x_{1}, x_{2}$. Also

$$
\begin{aligned}
z & \equiv z\left(x_{1}, x_{2}, y\right)=\frac{y^{2}}{\pi} \int_{R^{2}} \frac{z\left(x_{1}^{\prime}, x_{2}^{\prime}, 0\right) d x_{1}^{\prime} d x_{2}^{\prime}}{\left[\left(\mathbf{x}-\mathbf{x}^{\prime}\right)^{2}+y^{2}\right]^{2}}=\frac{y^{2}}{\pi} \int_{\mathcal{D}} \frac{d x_{1}^{\prime} d x_{2}^{\prime}}{\left[\left(\mathbf{x}-\mathbf{x}^{\prime}\right)^{2}+y^{2}\right]^{2}}, \\
V_{i} \equiv V_{i}\left(x_{1}, x_{2}, y\right) & =\frac{\epsilon_{i j}}{\pi} \int_{R^{2}} \frac{z\left(x_{1}^{\prime}, x_{2}^{\prime}, 0\right)\left(x_{j}-x_{j}^{\prime}\right) d x_{1}^{\prime} d x_{2}^{\prime}}{\left[\left(\mathbf{x}-\mathbf{x}^{\prime}\right)^{2}+y^{2}\right]^{2}}=\frac{\epsilon_{i j}}{\pi} \int_{\mathcal{D}} \frac{\left(x_{j}-x_{j}^{\prime}\right) d x_{1}^{\prime} d x_{2}^{\prime}}{\left[\left(\mathbf{x}-\mathbf{x}^{\prime}\right)^{2}+y^{2}\right]^{2}} .
\end{aligned}
$$

The 3-form fluxes and an axion-dilaton vanish.

Generically, a supergravity background (2.8) is time-dependent. A static solution arises whenever geometry has a second Killing vector. This is the case if $\mathcal{D}$ is rotationally invariant about the origin in $R^{2}$, as for the circular fermionic droplets, or a fermion configurations corresponding to concentric circles. In the former case (say zero temperature fermion configuration) an explicit solution is [1]

$$
\begin{aligned}
z\left(r, y ; r_{0}\right) & =\frac{r^{2}-r_{0}^{2}+y^{2}}{2 \sqrt{\left(r^{2}+r_{0}^{2}+y^{2}\right)-4 r^{2} r_{0}^{2}}}, \\
V_{\phi} & =-\frac{1}{2}\left(\frac{r^{2}+y^{2}+r_{0}^{2}}{\sqrt{\left(r^{2}+r_{0}^{2}+y^{2}\right)^{2}-4 r^{2} r_{0}^{2}}}-1\right),
\end{aligned}
$$

where $r_{0}$ is the radius of the fermion droplet, and $\{r, \phi\}$ are the polar coordinates in 
$x_{1}, x_{2}$ plane. With (2.10) and the change of variables [1]

$$
\begin{aligned}
& y=r_{0} \sinh \rho \sin \theta, \\
& r=r_{0} \cosh \rho \cos \theta, \\
& \psi=\phi+t,
\end{aligned}
$$

one recovers from (2.8) the standard $A d S_{5} \times S^{5}$ metric

$$
d s^{2}=r_{0}\left[-\cosh ^{2} \rho d t^{2}+\sinh ^{2} \rho d \Omega_{3}^{2}+d \rho^{2}+d \theta^{2}+\cos ^{2} \theta d \psi^{2}+\sin ^{2} \theta d \tilde{\Omega}_{3}^{2}\right]
$$

We now turn to thermal coarse-graining of LLM geometries. We propose that the equilibrium geometry corresponding to coarse-graining 1/2 BPS states of LLM at temperature ${ }^{5} T$ is an LLM supergravity background with the source $z\left(x_{1}, x_{2}, 0\right)$ in (2.1) identical to the configuration of the free fermion gas in the phase space $R^{2}$ at temperature $T$. As such, the resulting coarse-grained state is supersymmetric. At first sight this might look counterintuitive: how can supersymmetry be consistent with finite temperature? The resolution is simply that this is an artifact of the thermal averaging in 1/2 BPS sector of the Hilbert space: if all states in (grand)canonical ensemble are supersymmetric, the thermal state is supersymmetric as well. With above conjecture, it is straightforward to obtain the required geometry. We expect the resulting fermion equilibrium configuration $\mathcal{D}$ to be rotationally invariant, i.e., it is (at most) a set of concentric circles.

The number density of fermions in a phase space at temperature $T$ is given by the Fermi distribution

$$
d N=n(r) \frac{2 \pi r d r}{2 \pi \hbar} \equiv \frac{1}{e^{(\epsilon(r)-\mu) / T}+1} \frac{2 \pi r d r}{2 \pi \hbar}
$$

where $\mu$ is a chemical potential ${ }^{6}$, and a fermion energy is given by (2.4). Keeping the total number $N$ of fermions (or the 5 -form flux) fixed, determines the chemical potential

$$
N=\int d N=\int_{0}^{\infty} \frac{1}{e^{(\epsilon(r)-\mu) / T}+1} \frac{2 \pi r d r}{2 \pi \hbar}=T \ln \left(1+e^{\mu / T}\right)
$$

Or

$$
\mu=T \ln \left(e^{N / T}-1\right)
$$

\footnotetext{
${ }^{5}$ In units of $1 / R_{A d S_{5}}$.

${ }^{6}$ Not to be confused with the chemical potential conjugate to the $U(1)$ charge $J$.
} 
Notice that at small temperatures (degenerate fermi gas)

$$
\mu \approx N, \quad T \ll N .
$$

The phase space configuration of the finite temperature fermion gas is a set of concentric rings about the origin of the phase space with the 'width' of a ring of radius $r_{i}$ being equal $n\left(r_{i}\right) \triangle r_{i}$. The radial separation between two consecutive rings is $\left(1-n\left(r_{i}\right)\right) \triangle r_{i}$. These leads ${ }^{7}$ to the coarse-grained source function $z^{T}(r, y)$ as

$$
\begin{aligned}
z^{T}(r, y) & =\sum_{i}\left(z\left(r, y ; r_{i}+n\left(r_{i}\right) \triangle r_{i}\right)-z\left(r, y ; r_{i}\right)\right) \\
& \approx \sum_{i} \frac{\partial z\left(r, y ; r_{i}\right)}{\partial r_{i}} n\left(r_{i}\right) \triangle r_{i} \\
& =\int_{0}^{\infty} d x n(x) \frac{\partial z(r, y ; x)}{\partial x}
\end{aligned}
$$

where $z(r, y ; x)$ is given by $(2.10)$. Alternatively,

$$
z^{T}(r, y)=-\int_{0}^{\infty} d x z(r, y ; x) \frac{\partial n(x)}{\partial x} .
$$

From (2.18) we can immediately recover the zero temperature limit. Indeed, at $T=0$, $n(x)$ is a step function. Thus $\partial n(x) / \partial x$ is $-\delta\left(x-r_{0}^{(T=0)}\right)$, which leads to (2.10). We did not succeed in explicitly evaluating (2.17) for arbitrary temperature. Since geometry dual to $z^{T}$ (2.17) fermion configuration is an LLM 1/2 BPS state, it is singularity-free and does not have a horizon.

In the rest of this section we present coarse-grained geometry in the limit of vanishingly small temperature. The computation is a standard one in the statistical physics of the degenerate fermi gas [18]. Given that for an arbitrary smooth function $f(\epsilon)$ such that $I$ defined as

$$
I=\int_{0}^{\infty} \frac{f(\epsilon) d \epsilon}{e^{(\epsilon-\mu) / T}+1},
$$

converges, we have

$$
I=\int_{0}^{\mu} f(\epsilon) d \epsilon+\frac{\pi^{2}}{6} T^{2} f^{\prime}(\mu)+\mathcal{O}\left(T^{4}\right)
$$

and using change of variables ${ }^{8}(2.11)$ the resulting geometry can be written as

$$
d s^{2}=-g_{t t} d t^{2}+g_{\rho \rho}\left(d \rho^{2}+d \theta^{2}\right)+g_{\psi \psi} d \psi^{2}+g_{\Omega_{3} \Omega_{3}} d \Omega_{3}^{2}+g_{\tilde{\Omega}_{3} \tilde{\Omega}_{3}} d \tilde{\Omega}_{3}^{2},
$$

\footnotetext{
${ }^{7}$ Concentric configurations are also discussed in [1].

${ }^{8}$ For concentric ring distributions $d \psi \equiv d \phi-d t \frac{V_{\phi} y^{2}}{r^{2} z^{2} e^{2 G}-y^{2} V_{\phi}^{2}}$.
} 
where for $\rho>0$ and up to $\mathcal{O}\left(T^{4}\right)$ terms

$$
\begin{aligned}
& g_{t t}=r_{0} \cosh ^{2} \rho(1-\gamma \\
& \left.\times \frac{\left(3 \cos ^{2} \theta-1\right) \cosh ^{4} \rho+\left(3 \cos ^{4} \theta-6 \cos ^{2} \theta+2\right) \cosh ^{2} \rho+\cos ^{2} \theta\left(2-\cos ^{2} \theta\right)}{\left(\cosh ^{2} \rho-\cos ^{2} \theta\right)^{3}}\right), \\
& g_{\rho \rho}=r_{0}\left(1+\gamma\left(\cosh ^{2} \rho+\cos ^{2} \theta-2\right)\right. \\
& \left.\times \frac{\left(3 \cos ^{2} \theta-1\right) \cosh ^{4} \rho-\cos ^{2} \theta\left(4-3 \cos ^{2} \theta\right) \cosh ^{2} \rho-\cos ^{4} \theta}{\left(\cosh ^{2} \rho-\cos ^{2} \theta\right)^{4}}\right), \\
& g_{\psi \psi}=r_{0} \cos ^{2} \theta\left(1-\gamma \frac{1}{\left(\cosh ^{2} \rho-\cos ^{2} \theta\right)^{4}}\right. \\
& \times\left[\left(3-9 \cos ^{2} \theta\right) \cosh ^{6} \rho-\left(6 \cos ^{4} \theta-19 \cos ^{2} \theta+4\right) \cosh ^{4} \rho\right. \\
& \left.\left.+\cos ^{2} \theta\left(3 \cos ^{4} \theta+3 \cos ^{2} \theta-8\right) \cosh ^{2} \rho-\cos ^{6} \theta\right]\right) \text {, } \\
& g_{\Omega_{3} \Omega_{3}}=r_{0} \sinh ^{2} \rho(1-\gamma \\
& \left.\times \frac{\left(3 \cos ^{2} \theta-1\right) \cosh ^{4} \rho-\cos ^{2} \theta\left(4-3 \cos ^{2} \theta\right) \cosh ^{2} \rho-\cos ^{4} \theta}{\left(\cosh ^{2} \rho-\cos ^{2} \theta\right)^{3}}\right), \\
& g_{\tilde{\Omega}_{3} \tilde{\Omega}_{3}}=r_{0} \sin ^{2} \theta(1+\gamma \\
& \left.\times \frac{\left(3 \cos ^{2} \theta-1\right) \cosh ^{4} \rho-\cos ^{2} \theta\left(4-3 \cos ^{2} \theta\right) \cosh ^{2} \rho-\cos ^{4} \theta}{\left(\cosh ^{2} \rho-\cos ^{2} \theta\right)^{3}}\right),
\end{aligned}
$$

and

$$
d \psi \equiv d \phi+d t\left(1-\gamma \frac{2\left(\cos ^{2} \theta+\sinh ^{2} \rho\right) \cosh ^{2} \rho}{\left(\cosh ^{2} \rho-\cos ^{2} \theta\right)^{3}}\right) .
$$

In $(2.22),(2.23) r_{0}$ is given by $(2.5)$, also we introduced

$$
\gamma \equiv \frac{2 \pi^{2} T^{2} \hbar^{2}}{3 r_{0}^{4}}=\frac{\pi^{2} T^{2}}{6 N^{2}}
$$

\section{Conclusion}

In this paper we constructed supergravity background dual to thermal averaging over LLM geometries. The resulting geometry is singularity free. It does not have a horizon either. A similar prescription when implemented on the full Hilbert space of the type 
IIB string excitations in asymptotic $A d S_{5} \times S^{5}$ background is expected to produce an AdS-Schwarzschild black hole. It is interesting to explore whether this coarse-grained geometry exhibit thermal properties of the dual finite temperature free fermions. One specific question is whether the ADM mass of the thermal geometry agrees with the free fermion gas energy. In the absence of a horizon, how does the coarse-grained geometry encode the entropy of the free fermion gas?

\section{Acknowledgments}

I would like to thank I. Bena, E. Gorbar and V. Miransky for helpful conversations. Research at Perimeter Institute is supported in part by funds from NSERC of Canada. Additional support by an NSERC Discovery grant is gratefully acknowledged.

\section{References}

[1] H. Lin, O. Lunin and J. Maldacena, "Bubbling AdS space and 1/2 BPS geometries," arXiv:hep-th/0409174.

[2] J. M. Maldacena, "The large $N$ limit of superconformal field theories and supergravity," Adv. Theor. Math. Phys. 2, 231 (1998) [Int. J. Theor. Phys. 38, 1113 (1999)] [arXiv:hep-th/9711200].

[3] O. Lunin and S. D. Mathur, "Metric of the multiply wound rotating string," Nucl. Phys. B 610, 49 (2001) [arXiv:hep-th/0105136].

[4] O. Lunin and S. D. Mathur, "AdS/CFT duality and the black hole information paradox," Nucl. Phys. B 623, 342 (2002) [arXiv:hep-th/0109154].

[5] O. Lunin and S. D. Mathur, Phys. Rev. Lett. 88, 211303 (2002) [arXiv:hepth/0202072].

[6] O. Lunin, S. D. Mathur and A. Saxena, "What is the gravity dual of a chiral primary?," Nucl. Phys. B 655, 185 (2003) [arXiv:hep-th/0211292].

[7] S. D. Mathur, A. Saxena and Y. K. Srivastava, "Constructing 'hair' for the three charge hole," Nucl. Phys. B 680, 415 (2004) [arXiv:hep-th/0311092]. 
[8] H. Elvang, R. Emparan, D. Mateos and H. S. Reall, "A supersymmetric black ring," arXiv:hep-th/0407065.

[9] J. P. Gauntlett and J. B. Gutowski, "Concentric black rings," arXiv:hepth/0408010.

[10] J. P. Gauntlett and J. B. Gutowski, "General concentric black rings," arXiv:hepth/0408122.

[11] I. Bena and N. P. Warner, "One ring to rule them all ... and in the darkness bind them?," arXiv:hep-th/0408106.

[12] H. Elvang, R. Emparan, D. Mateos and H. S. Reall, "Supersymmetric black rings and three-charge supertubes," arXiv:hep-th/0408120.

[13] I. Bena and P. Kraus, "Microscopic description of black rings in AdS/CFT," arXiv:hep-th/0408186.

[14] S. Giusto and S. D. Mathur, "Geometry of D1-D5-P bound states," arXiv:hepth/0409067.

[15] O. Aharony, S. S. Gubser, J. M. Maldacena, H. Ooguri and Y. Oz, "Large N field theories, string theory and gravity," Phys. Rept. 323, 183 (2000) [arXiv:hepth/9905111].

[16] A. Buchel and L. A. Pando Zayas, "Hagedorn vs. Hawking-Page transition in string theory," Phys. Rev. D 68, 066012 (2003) [arXiv:hep-th/0305179].

[17] D. Berenstein, "A toy model for the AdS/CFT correspondence," JHEP 0407, 018 (2004) [arXiv:hep-th/0403110].

[18] L. D. Landau and E. M. Lifshits, "Statistical Physics, Part 1", izdatelstvo "Nauka", Moscow, 1976. 